\title{
Erratum to: Detecting fraudulent labeling of rice samples using computer vision and fuzzy knowledge
}

Tenvir Ali ${ }^{1}$ - Zeeshan Jhandhir ${ }^{1}$ - Awais Ahmad ${ }^{2}$.

Murad Khan ${ }^{2}$ - Arif Ali Khan ${ }^{3}$ - Gyu Sang Choi ${ }^{1}$

Published online: 13 April 2017

(C) Springer Science+Business Media New York 2017

\section{Erratum to: Multimed Tools Appl \\ DOI 10.1007/s11042-017-4472-9}

In the original publication, Fig. 3 contains wrong label. The authors would like to present the correct figure below.

p1 $\widehat{\sim}(\mathrm{i}-1, \mathrm{j}+1)$

p4 $(i-1, j)$

p6 $(i-1, j-1)$

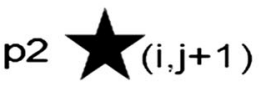

po $\curlyvee(i, j)$

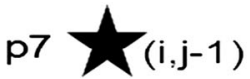

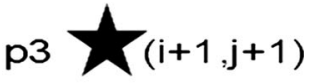

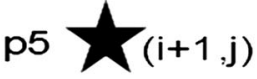

p8 $\widehat{Y}(\mathrm{i}+1, \mathrm{j}-1)$

The online version of the original article can be found at http://dx.doi.org/10.1007/s11042-017-4472-9

Gyu Sang Choi

castchoi@ynu.ac.kr

Tenvir Ali

tenvirali@ynu.ac.kr

Zeeshan Jhandhir

zeeshanjhandir@ynu.ac.kr

Awais Ahmad

awais.ahmad@live.com 
In the description of equation 19 "while here $\mathrm{K}$ is a constant" should be removed. The correct description is also presented below.

$$
o=f(s)=C \varphi\left(\sum_{j=1}^{N} w_{j} s_{j}-\theta\right) .
$$

In the Eq. 19, $\mathrm{C}$ is a constant and $\varphi$ represents a non-linear function whose parameter values are +1 and -1 (or 0 ). $\theta$ represents a threshold and $w j$ shows the weight.

Murad Khan

muradkhan23@gmail.com

Arif Ali Khan

aliakhan2-c@my.cityu.edu.hk

1 Department of Information and Communication Engineering, Yeungnam University, Gyeongbukdo, Republic of Korea

2 School of Computer Science and Engineering, Kyungpook National University, Daegu, Republic of Korea

3 Department of Computer Science, City University of Hong Kong, Kowloon, Hong Kong 